\title{
Automated Sub-ppm Mass Accuracy on an ESI-TOF for Use with Drug Discovery Compound Libraries
}

\author{
Justin G. Stroh, ${ }^{\text {a }}$ Christopher J. Petucci, ${ }^{a}$ Scott J. Brecker, ${ }^{\text {a }}$ \\ Nelson Huang, ${ }^{\text {b }}$ and James M. Lau ${ }^{\mathrm{c}}$ \\ ${ }^{a}$ Discovery Analytical Chemistry-Chemical Technologies, Chemical and Screening Sciences, Wyeth Research, \\ Collegeville, Pennsylvania, USA \\ ${ }^{b}$ Discovery Analytical Chemistry-Chemical Technologies, Chemical and Screening Sciences, Wyeth Research, \\ Cambridge, Massachusetts, USA \\ c Agilent Technologies, Wilmington, Delaware, USA
}

An automated, routine method to obtain sub-ppm accurate mass data on a benchtop electrospray ionization time-of-flight (ESI-TOF) mass spectrometer is described. Standards in the mass range 114 to $734 \mathrm{Da}$ were analyzed over a 5-day period to demonstrate intra- and interday precision and mean mass accuracy less than $1 \mathrm{ppm}$. One hundred drug discovery pharmaceutical compounds were used to demonstrate an absolute average mass accuracy of $0.47 \pm 0.31 \mathrm{ppm}$. This is in contrast to previous reports of accurate mass analysis using time-of-flight mass spectrometry (TOFMS) technology that operates within 3 to $5 \mathrm{ppm}$. The same 100 samples were also analyzed using Fourier transform mass spectrometry (FTMS) technology and yielded comparable results to the TOFMS analysis. (J Am Soc Mass Spectrom 2007, 18, 1612-1616) (c 2007 American Society for Mass Spectrometry

$\mathrm{T}$ The quality of drug discovery compound libraries is of significant interest to the pharmaceutical industry since these compounds will be used as potential candidates for future drugs [1, 2]. To assess the purity and integrity of these libraries, several analytical tests are performed, which can vary widely across the industry. The variation in testing may include analyses such as nuclear magnetic resonance (NMR), mass spectrometry, and high-performance liquid chromatography (HPLC) at one end to little more than the good word of the submitting chemist at the other. The need for high quality structural and purity data on compounds is most keenly felt when a compound coming out of the library gives excellent biological activity; however, upon re-synthesis of that compound, it is found that the proposed structure is completely inactive and the true structure is unknown. In the absence of high quality data, no firm conclusions can be drawn as to what the original compound was.

In terms of mass spectrometry, one of the highest quality pieces of information obtainable is accurate mass data to confirm the molecular formula of a compound. The importance of this information is well known [3] and has lead to the study of various ways to automate accurate mass data collection and analysis

Address reprint requests to Dr. J. G. Stroh, Discovery Analytical ChemistryChemical Technologies, Chemical and Screening Sciences, Wyeth Research, 500 Arcola Drive, Collegeville, PA 19426, USA. E-mail: strohj2@wyeth.com
[4-9]. An inter-laboratory comparison of techniques has shown in a controlled way the relative mass accuracy of various analyzers [10]. Four mass spectrometry analyzers that have been introduced as potential workhorses for this type of analysis are magnetic sector $[6,8]$, Fourier transform ion cyclotron resonance (FTMS) [7, 11], quadrupole time-of-flight (Q-TOF) [4, 9, 12, 13], and time-of-flight (TOF) $[14,15]$ mass analyzers. More recently, the Orbitrap [16, 17] has been demonstrated to perform exceptionally well as an accurate mass instrument.

Both magnetic sector and FTMS instruments give very high mass accuracy of $\approx 1 \mathrm{ppm}$ [10]. However, this mass accuracy advantage is offset by the fact that these instruments are difficult to run and maintain, requiring the support of a trained mass spectrometrist and the high cost of instrumentation. Further, until quite recently, FTMS with this accuracy was extremely difficult to couple with chromatography $[18,19]$. On the other hand, quadrupole TOF instruments are more readily available, less expensive, and easily coupled with chromatography, but of slightly lower mass accuracy as well (only $25 \%$ of measurements $\leq 1 \mathrm{ppm}$ in one study) [10, 20]. Finally, TOF instruments are the most widely available, least expensive, and easily coupled with chromatography, but suffer from the lowest published mass accuracy, usually at or about $\pm 5 \mathrm{ppm}[16]$ with an inter-laboratory comparison showing that $24 \%$ of measurements on TOF instruments yielding $\geq 10 \mathrm{ppm}$ mass 
accuracy [10]. Further, automation of accurate mass analyses using ESI-TOFMS has been difficult due to dead time effects on the time-to-digital conversion (TDC) circuitry that results in peak shifts to lower masses at high sample concentrations $[15,21]$.

Recently, Ferrer and Thurman reported [22] on an accurate mass ESI-TOF measurement of the mass of an electron by mass difference as 0.00062 Da with a standard deviation of $\pm 14.8 \% \quad(N=20)$. Unlike other TOFMS instruments that have been reported, the mass accuracy of this mass spectrometer does not suffer from dead time effects because it utilizes an analog-to-digital conversion circuit that counts ions continuously. This result is significant because it introduces the possibility that TOF technology may be used for routine, sub-ppm mass accuracy analysis that has only been demonstrated previously with FTMS and sector instruments [23]..$^{\circ}$ In $^{\circ}$ this $^{\circ}$ study $^{\circ}$ we $^{\circ}$ report $^{\circ}$ on $^{\circ}$ the $^{\circ}$ development ${ }^{\circ}$ and evaluation of a fully automated ESI-TOFMS method to obtain routine sub-ppm mass accuracy for compounds in drug discovery.

\section{Experimental}

\section{Materials}

Caffeine, 3-chloropyridine, warfarin, loperamide, reserpine, and erythromycin were purchased from SigmaAldrich (St. Louis, MO). A standard mixture was prepared by dissolving $0.1 \mathrm{mg}$ of each of these compounds into $1 \mathrm{~mL}$ of methanol. The medicinal chemistry department at Wyeth provided solutions of 100 different drug discovery compounds of $\sim 0.1$ to 2 $\mathrm{mg} / \mathrm{mL}$ in methanol.

\section{Liquid Chromatography}

An Agilent 1200 HPLC (Agilent Technologies, Palo Alto, CA) with a $3 \mathrm{~mm} \times 30 \mathrm{~mm}, 1.8 \mu \mathrm{m}$ SB-C18 column was used for chromatographic analysis before mass spectrometry. The chromatographic method consisted of a $1-\mathrm{min} 2.5 \mathrm{~mL} / \mathrm{min}$ gradient of $75 \%$ of $0.1 \%$ formic acid in water $/ 25 \%$ of $0.1 \%$ formic acid in acetonitrile to $5 \%$ of $0.1 \%$ of formic acid in water $/ 95 \%$ of $0.1 \%$ formic acid in acetonitrile with a hold for $0.25 \mathrm{~min}$ at the end of the gradient. For all experiments, samples were injected at $5 \mu \mathrm{L}$. The eluant from the HPLC column was sent through a photodiode array detector and then an active splitter at a split ratio of 100:1. The flow of the active splitter was set to $0.5 \mathrm{~mL} / \mathrm{min}$ of $50 / 50$ methanol/ water, which was directed into the inlet of the electrospray probe of the mass spectrometer.

\section{Mass Spectrometry}

TOFMS. An Agilent TOF mass spectrometer (Agilent Technologies, Palo Alto, CA) equipped with an electrospray probe was used for accurate mass measurements. The mass spectrometer was operated in positive ion mode with a capillary voltage of $3000 \mathrm{~V}$, nebulizer of 40 psig, drying gas of $8 \mathrm{~L} / \mathrm{min}$, gas temperature of $300^{\circ} \mathrm{C}$, fragmentor of $180 \mathrm{~V}$, skimmer of $60 \mathrm{~V}$, OCT DC1 of $37 \mathrm{~V}$, and OCT RFV of $250 \mathrm{~V}$. Mass spectra were collected at a rate of 4 spectra/s and 3284 transients / spectrum over a mass range 10 to $1000 \mathrm{Da}$. The multichannel plate detector voltage was $650 \mathrm{~V}$ and the photomultiplier tube voltage was $696 \mathrm{~V}$. The pulser was set to a pulse width of 125 counts/pulse with a pulse width of 25 counts. A second reference sprayer orthogonal to the sample sprayer in the electrospray source was used to introduce a reference solution for accurate mass determinations. The reference mass ions of $\mathrm{m} / \mathrm{z}$ 121.0509 and 922.0098 (resolution FWHM of 11,000 at $m / z$ 922.0098) had counts of 140,000 and 30,000, respectively. The detection window for the reference masses was set to 50 ppm with an average of 10 scans and a minimum peak height of 100 counts/s. A mass calibration was performed with an Agilent tune mix from 100 to $1600 \mathrm{Da}$.

FTMS. The FTMS instrument used in this study was a Bruker APEX III 7 Tesla FTMS equipped with an Apollo II electrospray source. An Agilent 1100 HPLC was used to infuse samples to the FTMS at a flow rate of 0.15 $\mathrm{mL} / \mathrm{min}$. The solvent flow was further reduced by a passive splitter to direct $\sim 30 \%$ of the flow to the mass spectrometer. The rest of the flow was directed to waste. The HPLC solvent was 1:1 water:acetonitrile with $0.1 \%$ formic acid. The autosampler was programmed to perform stacked injections, which produced a data acquisition rate of about one sample $/ \mathrm{min}$. Each mass spectrum was typically the sum of 10 or 15 scans. External calibration of the FTMS was performed with a calibration mixture commercially available from Agilent Technologies with data acquisition carried out immediately after calibration.

\section{Data Processing}

A computer algorithm provided with the Agilent TOFMS software was used to automatically process accurate mass data. Accurate masses were determined by averaging 5 spectra across the peak in the total ion chromatogram with a mass spectral threshold of $30 \%$ of the detector saturation threshold ( $~ 500,000$ counts) and a minimum threshold of 100 counts.

FTMS data acquisition was controlled by XMASS (Bruker Daltonics). The software for automated data acquisition and ${ }^{\circ}$ processing ${ }^{\circ}{ }^{\circ}{ }^{\circ}$ been $^{\circ}$ described $^{\circ}$ previously $^{\circ}[7]$.

\section{Results and Discussion}

The TOFMS described above was initially tested for intra- and interday precision and accuracy over a 5-day period $^{\circ}$ using $^{\circ} \mathrm{a}^{\circ}$ mixture $^{\circ}$ of $^{\circ} \operatorname{six}^{\circ} \operatorname{compounds}^{\circ}\left(\right.$ Table $^{\circ} 1$, Table ${ }^{\circ}$, ${ }^{\circ}$ and ${ }^{\circ}$ Table $\left.^{\circ} 3\right)^{\circ}$ ranging ${ }^{\circ}$ from ${ }^{\circ} 114^{\circ}$ to ${ }^{\circ} 734^{\circ} \mathrm{Da} .^{\circ}$ This mass range is what one might reasonably expect to find in a pharmaceutical company's small molecule library. 
Table 1. Experimental accurate masses for the protonated molecular ions of standard drug compounds

\begin{tabular}{llccrr}
\hline Compound & \multicolumn{1}{c}{ Formula } & $\begin{array}{c}\text { Theoretical } \\
\text { mass }\end{array}$ & $\begin{array}{c}\text { Day 1 } \\
\text { mass avg }\end{array}$ & $\begin{array}{c}\text { Day 3 } \\
\text { mass avg }\end{array}$ & $\begin{array}{c}\text { Day } 5^{\text {a }} \\
\text { mass avg }\end{array}$ \\
\hline \hline 3-Chloropyridine & C5H5CIN & 114.01050 & 114.01043 & 114.01049 & 114.01047 \\
Caffeine & C8H11N4O2 & 195.08765 & 195.08781 & 195.08769 & 195.08774 \\
Warfarin & C19H17O4 & 309.11214 & 309.11233 & 309.11203 & 309.11203 \\
Loperamide & C29H34CIN2O2 & 477.23033 & 477.23057 & 477.23040 & 477.23044 \\
Reserpine & C33H41N2O9 & 609.28066 & 609.28111 & 609.28103 & 609.28110 \\
Erythromycin & C37H68NO13 & 734.46852 & 734.46892 & 734.46867 & 734.46879 \\
\hline
\end{tabular}

${ }^{a}$ Data for Days 1,3 , and 5 are the averages $(n=5)$ of experimental accurate masses.

Each compound's average ppm error and standard deviation was based on a total of 15 mass measurements 9 taken ${ }^{\circ}{ }^{\circ}{ }^{\circ}$ \%5-day period 9 Table 1 ). ${ }^{\circ}$ On Days 1,3 , and 5 , the mass of each compound was measured five times.

Table $^{\circ} 1^{\circ}$ shows ${ }^{\circ}$ the ${ }^{\circ}$ average ${ }^{\circ}$ observed ${ }^{\circ}$ masses ${ }^{\circ}$ for ${ }^{\circ}$ each of six compounds listed over a 5-day period compared with the theoretical mass for each compound. The data in this table clearly demonstrate that there is no bias in the system relating to the molecular weight of the analyte for small molecule analysis. Of particular note in Table 29 's that the intraday precision and accuracy for the ppm errors of each of the standard compounds for Days 1, 3, and 5 was less than 1 ppm. The smallest average ppm error was $0.13 \pm 0.29 \mathrm{ppm}$ for loperamide on Day 3 and the largest average ppm error was $0.79 \pm$ $0.13 \mathrm{ppm}$ for caffeine on Day 1 . The highest mass accuracy obtained for the entire dataset was $0.04 \mathrm{ppm}$ for loperamide on Day 5. Further, the highest single measured mass error of any of these compounds throughout the entire 5-day period was $1.64 \mathrm{ppm}$, for reserpine on Day 5 (data not shown). In addition, the interday precision and accuracy was less than $1 \mathrm{ppm}$ for the ppm errors of each of the standard compounds for Days 1, 3, and 5, demonstrating the excellent ruggedness ${ }^{\circ}$ of $^{\circ}$ this ${ }^{\circ} \mathrm{TOF}^{\circ}$ mass $^{\circ}$ spectrometer $^{\circ}\left(\right.$ Table $\left.^{\circ} 3\right)$.

The data discussed above clearly demonstrate that sub-ppm mass accuracy is routinely attainable using this instrumentation. However, a problem with clearly defined standards such as those shown here is that they often do not fully represent the range of issues that are normally observed with drug discovery compounds, which lead to poor mass accuracy such as concentration, solubility, and stability of the analyte to name a few. In our view, by far the worst of these issues is submission by synthetic chemists of a wide range of sample concentrations, usually at the high concentration end. While analysis of samples at a few $\mathrm{mg} / \mathrm{mL}$ is appropriate for NMR and HPLC, this concentration is too high for most mass spectrometry applications and especially for accurate mass. Since a truly automated accurate mass system was desired, the synthetic chemists were permitted to submit samples at their normal concentrations $(1-10 \mathrm{mg} / \mathrm{mL})$ with dilution being performed within the instrumentation. Consequently, we have developed an LC/MS system that injects samples at normal HPLC concentrations $(\mathrm{mg} / \mathrm{mL})$ with HPLC analysis via diode array detection. Post-UV analysis, a dynamic splitter with a make-up isocratic pump has been inserted, which allows us to dilute the sample between the UV detector and the mass spectrometer, bringing the sample into a concentration range $(\mathrm{ng} / \mathrm{mL})$ that is most suitable for mass spectrometry. Since electrospray ionization is a concentration-sensitive technique, it is not appropriate to simply split the eluant between the UV detector and the mass spectrometer, so a dilution must be performed. Analytes are diluted between a factor of 100 and 1000 such that the solvent composition $\left(50 / 50 \mathrm{H}_{2} \mathrm{O}: \mathrm{MeOH}\right)$ actually flowing into the mass spectrometer remains virtually constant over the course of a gradient HPLC run since $99 \%$ to $99.9 \%$ of the solvent is coming from the isocratic pump. To demonstrate the utility of this automated accurate mass LC/MS system, the mass accuracy of 100 drug discovery compounds of unknown concentration were determined before submission into Wyeth's compound library. The mass range of this particular dataset was 255 to 709 Da with a median average mass of $430 \mathrm{Da}$. All samples were taken from sample injection through data analysis and processing without human intervention. The observed mass errors for these 100 compounds are shown $^{\circ}$ in $^{\circ}$ Figure $^{\circ} 1 .^{\circ}$ The $^{\circ}$ highest $^{\circ}$ single $^{\circ}$ mass $^{\circ}$ error

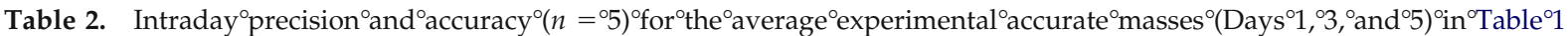

\begin{tabular}{|c|c|c|c|c|c|c|}
\hline Compound & $\begin{array}{c}\text { Day } 1 \\
\text { ppm avg }\end{array}$ & Std dev & $\begin{array}{c}\text { Day } 3 \\
\text { ppm avg }\end{array}$ & Std dev & $\begin{array}{c}\text { Day } 5 \\
\text { ppm avg }\end{array}$ & Std dev \\
\hline 3-Chloropyridine & -0.60 & 0.38 & -0.15 & 0.49 & -0.27 & 0.49 \\
\hline Caffeine & 0.79 & 0.13 & 0.22 & 0.27 & 0.48 & 0.28 \\
\hline Warfarin & 0.63 & 0.38 & -0.34 & 0.41 & -0.35 & 0.21 \\
\hline Loperamide & 0.49 & 0.40 & 0.13 & 0.29 & 0.22 & 0.32 \\
\hline Reserpine & 0.73 & 0.28 & 0.62 & 0.63 & 0.73 & 0.67 \\
\hline Erythromycin & 0.54 & 0.27 & 0.21 & 0.23 & 0.37 & 0.33 \\
\hline
\end{tabular}


observed was $1.3 \mathrm{ppm}$, and $92 \%$ of the samples gave a mass error below $1 \mathrm{ppm}$. The absolute mean average mass error was $0.47 \pm 0.31 \mathrm{ppm}$, demonstrating that sub-ppm mass accuracies are routinely obtainable using a TOF mass spectrometer.

Routine sub-ppm mass accuracies as demonstrated here have not been previously attainable using TOF technology. Previously, TOF technology mass accuracy was limited to the 5-ppm range whereas sub-ppm mass accuracy was the exclusive domain of FTMS and sector technolog $y^{\circ}[10] . .^{\circ}$ Therefore, ${ }^{\circ}$ for ${ }^{\circ}$ comparison $^{\circ}$ purposes, the mass accuracies of the same 100 drug discovery compounds in the same vials used for the TOFMS analysis were determined using an automated FTMS with external calibration. We believe that external calibration more closely represents what is available with a truly automated system than internal calibration using FTMS technology since for internal calibration, calibrant and analyte must be relatively balanced, which would require human intervention, thereby negating automation. Under these conditions, the observed average mass error was $1.37 \pm 0.90 \mathrm{ppm}$. This value is in substantial agreement with what is found in the literature $\left[5,{ }^{\circ} 10,{ }^{\circ} 24\right]^{\circ}$ for $^{\circ}$ mass $^{\circ}$ measurements ${ }^{\circ}$ via $^{\circ} \mathrm{FTMS}^{\circ}$ with external calibration with mass errors ranging from 0.5 to $1.75 \mathrm{ppm}$ with standard deviations of approximately ${ }^{\circ} \pm 9.5^{\circ} \mathrm{ppm}^{\circ}[24] .{ }^{\circ}{ }^{\circ}{ }^{\circ}$ addition, ${ }^{\circ}$ mass ${ }^{\circ}$ accuracies $<0.5 \mathrm{ppm}$ have been reported using internal calibration with controlled sample concentrations to minimize space $^{\circ}$ charging ${ }^{\circ}[24]$.

\section{Conclusions}

An ESI-TOF instrument has been demonstrated to produce routine sub-ppm mass accuracies (0.47 \pm $0.31 \mathrm{ppm}$ ) using drug discovery compounds. The literature to date has shown that mass accuracies of this caliber are possible only by using FTMS technology. However, it is now possible to routinely achieve this type of mass accuracy using simple TOFMS technology. Given the simplicity of the TOFMS design and automation, it should now be possible for

Table 3. Interday precision and accuracy $(n=15)$ for the average of experimental accurate masses (Days 1,3, and 5) in Table 2

\begin{tabular}{lrc}
\hline Compound & $\mathrm{ppm}$ & Std dev \\
\hline \hline 3-Chloropyridine & -0.34 & 0.23 \\
Caffeine & 0.49 & 0.29 \\
Warfarin & -0.02 & 0.56 \\
Loperamide & 0.28 & 0.18 \\
Reserpine & 0.69 & 0.06 \\
Erythromycin & 0.37 & 0.17 \\
\hline
\end{tabular}

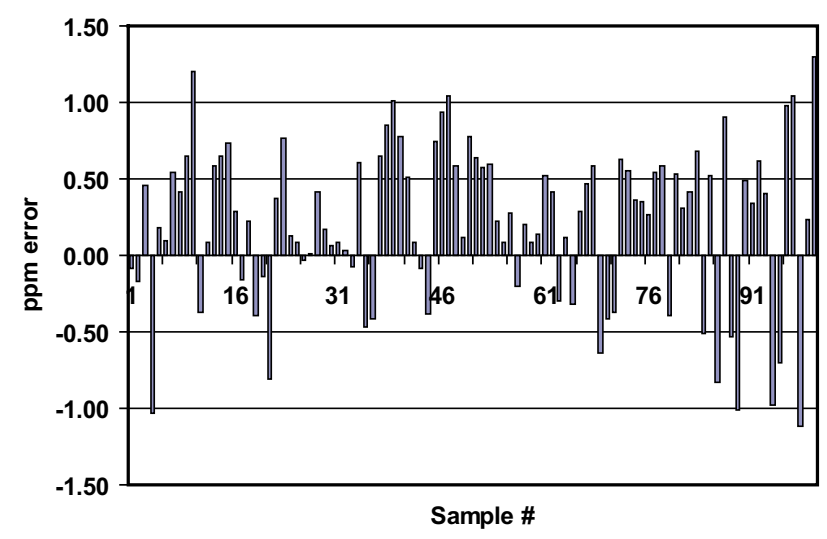

Figure 1. Distribution of mass accuracies (ppm) or 100 drug discovery library compounds (singletons).

sub-ppm mass accuracies to be obtained routinely in most mass spectrometry laboratories.

\section{References}

1. Dulery, B. D.; Verne-Mismer, J.; Wolf, E.; Kugel, C.; Van Hijfte, L. Analyses of Compound Libraries Obtained by High-Throughput Parallel Synthesis: Strategy of Quality Control by High-Performance Liquid Chromatography, Mass Spectrometry, and Nuclear Magnetic Resonance Techniques. J. Chromatogr. B Biomed. Sci. App. 1999, 725(1), 39-47.

2. Bindseil, K. U.; Jakupovic, J.; Wolf, D.; Lavayre, J.; Leboul, J.; van der Pyl, D. Pure Compound Libraries; A New Perspective for Natural Product Based Drug Discovery. Drug Discov. Today 2001, 6(16), 840-847.

3. Gross, M. L. Accurate Masses for Structure Confirmation. J. Am. Soc. Mass Spectrom. 1994, 5(2), 57.

4. Colombo, M.; Sirtori F. R.; Rizzo, V. A Fully Automated Method for Accurate Mass Determination Using High-Performance Liquid Chromatography with a Quadrupole/Orthogonal Acceleration Time-ofFlight Mass Spectrometer. Rapid Commun. Mass Spectrom. 2004, 18(4), 511-517.

5. Dykes, S.; Fancy, S.-A.; Perkins, G. L.; Pullen, F. S. The Automation of a Commercial Fourier Transform Mass Spectrometer to Provide a Quick and Robust Method for Determining Exact Mass for the Synthetic Chemist. Eur. J. Mass Spectrom. 2003, 9(2), 73-80.

6. Haas, M. J. Fully Automated Exact Mass Measurements by HighResolution Electrospray Ionization on a Sector Instrument. Rapid Commun. Mass Spectrom. 1999, 13(5), 381-383.

7. Huang, N; Siegel, M. M.; Kruppa, G. H.; Laukien, F. H. Automation of a Fourier Transform Ion Cyclotron Resonance Mass Spectrometer for Acquisition, Analysis, and E-mailing of High-Resolution Exact-Mass Electrospray Ionization Mass Spectral Data. J. Am. Soc. Mass Spectrom. 1999, 10(11), 1166-1173.

8. Perkins, G.; Pullen, F.; Thompson, C. Automated High Resolution Mass Spectrometry for the Synthetic Chemist. J. Am. Soc. Mass Spectrom. 1999, 10(6), 546-551.

9. Thomas, S. R.; Gerhard, U. Open-Access High-Resolution Mass Spectrometry in Early Drug Discovery. J. Mass Spectrom. 2004, 39(8), 942-948.

10. Bristow, A. W. T.; Webb, K. S. Intercomparison Study on Accurate Mass Measurement of Small Molecules in Mass Spectrometry. J. Am. Soc. Mass Spectrom. 2003, 14(10), 1086-1098.

11. Thompson, C. M; Richards, D. S.; Fancy, S.-A.; Perkins, G. L.; Pullen, F. S.; Thom, C. A Comparison of Accurate Mass Techniques for the Structural Elucidation of Fluconazole. Rapid Commun. Mass Spectrom. 2003, 17(24), 2804-2808.

12. Williams, J. P; Lock, R.; Patel, V. J.; Scrivens, J. H. Polarity Switching Accurate Mass Measurement of Pharmaceutical Samples Using Desorption Electrospray Ionization and a Dual Ion Source Interfaced to an Orthogonal Acceleration Time-of-Flight Mass Spectrometer. Anal. Chem. 2006, 78(21), 7440-7445.

13. Wolff, J.-C.; Fuentes, T. R.; Taylor, J. Investigations into the accuracy and precision obtainable on accurate mass measurements on a quadrupole orthogonal acceleration time-of-flight mass spectrometer using liquid chromatography as sample introduction. Rapid Commun. Mass Spectrom. 2003, 17(11), 1216-1219.

14. Petucci, C.; Mallis, L. Automated Accurate Mass Data Processing Using a Gas Chromatograph/Time-of-Flight Mass Spectrometer in Drug Discovery. Rapid Commun. Mass Spectrom. 2005, 19(11), 1492-1498.

15. Wu, J.; McAllister, H. Exact Mass Measurement on an Electrospray Ionization Time-of-Flight Mass Spectrometer: Error Distribution and Selective Averaging. J. Mass Spectrom. 2003, 38(10), 1043-1053. 
16. Touber, M. E.; Van Engelen, M. C.; Georgakopoulus, C.; Van Rhijn, J. A.; Nielen, M. W. F. Multidetection of Corticosteroids in Sports Doping and Veterinary Control Using High Resolution Liquid Chromatography/ Time-of-Flight Mass Spectrometry. Anal. Chim. Acta 2007, 586(1/2), 137-146

17. Hu, Q; Noll, R. J.; Li, H.; Makarov, A.; Hardman, M.; Cooks, R. G. The Orbitrap: A new mass spectrometer. J. Mass Spectrom. 2005, 40(4), $430-443$.

18. O'Connor, P. B; Pittman, J. L.; Thomson, B. A.; Budnik, B. A.; Cournoyer, J. C.; Jebanathirajah, J.; Lin, C.; Moyer, S.; Zhao, C. A new hybrid electrospray Fourier transform mass spectrometer: Design and performance characteristics. Rapid Commun. Mass Spectrom. 2005, 20(2), $259-266$.

19. Syka, J. E. P; Marto, J. A.; Bai, D. L.; Horning, S.; Senko, M. W.; Schwartz, J. C.; Ueberheide, B.; Garcia, B.; Busby, S.; Muratore, T.; Shabanowitz, J.; Hunt, D. F. Novel Linear Quadrupole Ion Trap/FT Mass Spectrometer: Performance Characterization and Use in the Comparative Analysis of
Histone H3 Post-Translational Modifications. J. Proteome Res. 2004, 3(3), 621-626.

20. Ferrer, I.; Thurman, E. M.; Fernandez-Alba, A. R. Quantitation and Accurate Mass Analysis of Pesticides in Vegetables by LC/TOF-MS. Anal. Chem. 2005, 77(9), 2818-2825.

21. Koefeler, H. C.; Gross, M. L. Correction of Accurate Mass Measurement for Target Compound Verification by Quadrupole Time-of-Flight Mass Spectrometry. J. Am. Soc. Mass Spectrom. 2005, 16(3), 406-408.

22. Ferrer, I.; Thurman, E. M. Measuring the Mass of an Electron by LC/TOFMS: A Study of "Twin Ions." Anal. Chem. 2005, 77(10), 3394-3400.

23. Bristow, A. W. T. Accurate Mass Measurement for the Determination of Elemental Formula-A Tutorial. Mass Spectrom. Rev. 2006, 25(1), 99-111.

24. Herniman, J. M; Langley, G. J.; Bristow, T. W. T.; O'Connor, G. The Validation of Exact Mass Measurements for Small Molecules Using FT-ICRMS for Improved Confidence in the Selection of Elemental Formulas. J. Am. Soc. Mass Spectrom. 2005, 16(7), 1100-1108. 